\title{
A FIXED-POINT MINIMUM ERROR ENTROPY ALGORITHM
}

\author{
Seungju Han, Jose Principe
}

\section{CNEL, Department of Electrical and Computer Engineering, University of Florida, Gainesville, USA}

\begin{abstract}
In this paper, we propose the Fixed-Point Minimum Error Entropy (Fixed-Point MEE) as an alternative to the Minimum Error Entropy (MEE) algorithm for training adaptive systems. The fixed-point algorithms are different from the gradient methods like MEE, and are proven to be faster, more stable and step-size free. This characteristic is due to the second order update similar to Recursive LeastSquares (RLS) that tracks the Wiener solution with every update. We study the effect of design parameters, namely the forgetting factor, the window length, and the kernel size, on the convergence properties of the newly introduced recursive Fixed-Point MEE. Also, we test the performance of both the algorithms for two classic problems of system identification. Finally, we conclude that the Fixed-Point MEE performs better than MEE.
\end{abstract}

\section{INTRODUCTION}

Information Theoretic Learning was made possible with the introduction of smooth sample estimators of entropy as proposed by Principe and collaborators [1],[2]. The nonparametric entropy estimator is based on Renyi's quadratic entropy, which can be applied directly to data collected from experiments and without imposing any a priori assumptions about the underlying probability density function (PDF). The goal of entropy in supervised learning follows the MSE framework. Given a set of input-desired signal pairs, the entropy of the error over the training dataset is minimized. The procedure is called Minimum Error Entropy (MEE) [3].

The MEE algorithm is based on simple gradient techniques, so it requires a priori knowledge of the input process statistics (power and dynamic range) to select the learning rate $\mu$ for stability and convergence. Since this knowledge is usually unavailable, the step size is normally estimated prior to the beginning the adaptation process for a given misadjustment or speed of convergence.

Recently, there has been a growing interest in fixed-point algorithms. These algorithms are different from the gradient methods and are proven to be faster, more stable and stepsize free. Fixed-Point algorithms in neural learning theory have received considerable attention due to their properties of low computational requirement and fast iteration convergence [4],[5]. In the fixed-point algorithms, the computations are made in batch mode, i.e., a large number of data points are used in a single step of the algorithm. In particular, they are parallel, distributed, computationally simple, and require little memory space.

The purpose of this paper is to propose an enhanced MEE training method based on the fixed-point algorithm, which is referred as the Fixed-Point Minimum Error Entropy (Fixed-Point MEE) algorithm. Since there is a need to achieve smooth and fast convergence to the optimal solution using low-complexity learning rules in on-line adaptation, our desire is to come up with a recursive Fixed-Point MEE algorithm. This algorithm, possibly using a forgetting factor, will preserve some information from the past, while updating the weight based on the newly acquired samples.

The paper is organized as follows. Section 2 summarizes the concept of MEE. We derive the Fixed-Point MEE in section 3 and modify to the recursive Fixed-Point MEE in section 4 . Section 5 deals with simulation results and finally we conclude in section 6 .

\section{SUMMARY OF MINIMUM ERROR ENTROPY}

Suppose that the adaptive system is an FIR structure with a weight vector $\mathbf{w}=\left[w_{1}, w_{2}, \ldots, w_{M}\right]^{T}$. The instantaneous error is $e_{k}=d_{k}-\mathbf{w}_{k}{ }^{T} \mathbf{u}_{k}$, where $d_{k}$ is the desired response, and $\mathbf{u}_{k}$ is the input vector at time $k$. The error PDF at a point $e$ is estimated using Parzen window estimation with a kernel function as

$$
\hat{f}_{e}(e)=\frac{1}{N} \sum_{i=1}^{N} K_{\sigma}\left(e-e_{i}\right)
$$

where $K_{\sigma}(\cdot)$ is the Gaussian kernel function with a kernel size $\sigma$. So, Renyi's quadratic entropy estimator for a set of discrete data samples becomes [1]:

$$
\begin{gathered}
H_{R 2}(e)=-\log \int \hat{f}_{e}^{2}(e) d e=-\log V(e) \\
V(e)=\frac{1}{N^{2}} \sum_{i=1}^{N} \sum_{j=1}^{N} K_{\sigma \sqrt{2}}\left(e_{j}-e_{i}\right) \leq V(0)
\end{gathered}
$$


The argument of the 'log' in (3) is named the information potential $V(e)$ due to the similarity between this quantity and the physical potential energy of an ensemble of particles. Minimizing the entropy $H_{R 2}(e)$ is equivalent to maximizing the information potential $V(e)$ since the $\log$ is a monotonic function.

The information potential estimate at time $k$, denoted by $V_{k}(e)$ is given by

$$
V_{k}(e)=\frac{1}{k^{2}} \sum_{i=1}^{k} \sum_{j=1}^{k} K_{\sigma \sqrt{2}}\left(e_{j}-e_{i}\right)
$$

The forgetting recursive entropy estimator updates the information potential according to [6]

$$
V_{k+1}(e)=(1-\lambda) V_{k}(e)+\frac{\lambda}{L} \sum_{i=k-L+1}^{k} K_{\sigma \sqrt{2}}\left(e_{k+1}-e_{i}\right)
$$

where $\lambda$ and $L$ are called the forgetting factor and window length. The weights of the filter are adapted to minimize the error entropy using gradient descent. Since minimizing the entropy $H_{R 2}(e)$ is equivalent to maximizing the information potential $V(e)$, the following update rule can be employed:

$$
\mathbf{w}_{k+1}=\mathbf{w}_{k}+\mu \frac{\partial V_{k+1}(e)}{\partial \mathbf{w}}
$$

where the gradient will be updated which in this case corresponds to

$$
\begin{aligned}
\frac{\partial V_{k+1}(e)}{\partial \mathbf{w}}=(1-\lambda) \frac{\partial V_{k}(e)}{\partial \mathbf{w}}+ & \\
& \frac{\lambda}{L} \sum_{i=k-L+1}^{k}\left[e_{k+1}-e_{i}\right] \cdot K_{\sigma \sqrt{2}}\left(e_{k+1}-e_{i}\right) \cdot\left[\frac{\partial y_{k+1}}{\partial \mathbf{w}}-\frac{\partial y_{i}}{\partial \mathbf{w}}\right]
\end{aligned}
$$

Notice that if $\lambda=1$, then this recursive gradient reduces to the stochastic information gradient (SIG) [7].

The above mentioned algorithm is based on gradient methods and they involve the selection of right step-sizes to ensure convergence. In general, the step-sizes have an upper bound that is a function of the eigenvalues of the input data. This fact makes it very hard on many occasions to choose the proper step-size. In next section, we will present a fixedpoint MEE algorithm, which is on-line, step-size free, and faster than the gradient method.

\section{FIXED-POINT MINIMUM ERROR ENTROPY}

Given the criterion $V(e)$, and without assuming any prior knowledge about the location of its minimizing argument $\mathbf{w}_{*}$, we wish to devise a procedure that starts from an initial guess for $\mathbf{w}_{*}$ and then improves upon it in a recursive manner until ultimately converging to $\mathbf{w}_{*}$. Using the first order optimality condition, we find the stationary point $\mathbf{w}$ as

$\frac{\partial V(e)}{\partial \mathbf{w}}=\frac{1}{2 N^{2} \sigma^{2}} \sum_{i=1}^{N} \sum_{j=1}^{N}\left[e_{j}-e_{i}\right] \cdot K_{\sigma \sqrt{2}}\left(e_{j}-e_{i}\right) \cdot\left[\frac{\partial y_{j}}{\partial \mathbf{w}}-\frac{\partial y_{i}}{\partial \mathbf{w}}\right]=0$

where $e_{k}=d_{k}-y_{k}=d_{k}-\mathbf{w}_{k}{ }^{T} \mathbf{u}_{k}$. Changing (8) to the fixed point form, we get

$$
\begin{aligned}
\mathbf{w}_{*}= & f\left(\mathbf{w}_{*}\right) \\
= & \left(\frac{1}{N^{2}} \sum_{i=1}^{N} \sum_{j=1}^{N} K_{\sigma \sqrt{2}}\left(e_{j}-e_{i}\right) \cdot\left[\mathbf{u}_{j}-\mathbf{u}_{i}\right] \cdot\left[\mathbf{u}_{j}-\mathbf{u}_{i}\right]^{T}\right)^{-1} \\
& \cdot\left(\frac{1}{N^{2}} \sum_{i=1}^{N} \sum_{j=1}^{N} K_{\sigma \sqrt{2}}\left(e_{j}-e_{i}\right) \cdot\left[d_{j}-d_{i}\right] \cdot\left[\mathbf{u}_{j}-\mathbf{u}_{i}\right]\right) .
\end{aligned}
$$

Equation (9) is the basis of our iteration algorithm. Let the weight vector $\mathbf{w}_{k}$ at iteration $k$ be the estimate of an optimal solution. Then, the estimate of the new weight vector at iteration $k+1$ according to (9) is,

$$
\begin{aligned}
\mathbf{w}_{k+1}= & F\left(\mathbf{w}_{k}\right) \\
= & \left(\frac{1}{k^{2}} \sum_{i=1}^{k} \sum_{j=1}^{k} K_{\sigma \sqrt{2}}\left(e_{j}-e_{i}\right) \cdot\left[\mathbf{u}_{j}-\mathbf{u}_{i}\right] \cdot\left[\mathbf{u}_{j}-\mathbf{u}_{i}\right]^{T}\right)^{-1} \\
& \cdot\left(\frac{1}{k^{2}} \sum_{i=1}^{k} \sum_{j=1}^{k} K_{\sigma \sqrt{2}}\left(e_{j}-e_{i}\right) \cdot\left[d_{j}-d_{i}\right] \cdot\left[\mathbf{u}_{j}-\mathbf{u}_{i}\right]\right) \\
= & \mathbf{R}_{E}\left(\mathbf{w}_{k}\right)^{-1} \mathbf{P}_{E}\left(\mathbf{w}_{k}\right)
\end{aligned}
$$

where $\mathbf{R}_{E}\left(\mathbf{w}_{k}\right)$ and $\mathbf{P}_{E}\left(\mathbf{w}_{k}\right)$ are the pairwise difference autocorrelation and crosscorrelation of the input and desired signals, weighted by the error kernels derived from the entropy formulation. We will call them respectively the entropy weighted delta autocorrelation (ER) matrix and entropy weighted delta crosscorrelation (EP) vector,

$$
\begin{aligned}
& \mathbf{R}_{E}\left(\mathbf{w}_{k}\right)=\frac{1}{k^{2}} \sum_{i=1}^{k} \sum_{j=1}^{k} K_{\sigma \sqrt{2}}\left(e_{j}-e_{i}\right) \cdot\left[\mathbf{u}_{j}-\mathbf{u}_{i}\right] \cdot\left[\mathbf{u}_{j}-\mathbf{u}_{i}\right]^{T} \\
& \mathbf{P}_{E}\left(\mathbf{w}_{k}\right)=\frac{1}{k^{2}} \sum_{i=1}^{k} \sum_{j=1}^{k} K_{\sigma \sqrt{2}}\left(e_{j}-e_{i}\right) \cdot\left[d_{j}-d_{i}\right] \cdot\left[\mathbf{u}_{j}-\mathbf{u}_{i}\right] \cdot
\end{aligned}
$$

This is analogous to the RLS update rule that tracks the Wiener solution with every update [8].

A standard method for proving the convergence of a fixed-point algorithm is the well-known BanachCaccioppoli contraction theorem [9], which ensures that in, a complete metric space, a fixed-point algorithm based on a 
contractive operator surely converges to a steady state. In this case, the iteration is carried out on a $L_{\mathrm{w}}$-dimensional hyper-sphere that is a complete metrizable space, and the fixed-point operator $F\left(\mathbf{w}_{k}\right)$ is apparently differentiable. As a consequence, when

$$
\left\|\frac{\partial F\left(\mathbf{w}_{k}\right)}{\partial \mathbf{w}_{k}}\right\|<1
$$

is satisfied at every iteration, the convergence of the proposed Fixed-Point MEE learning rule is guaranteed.

\section{RECURSIVE FIXED-POINT MEE}

Investigating the structure of the estimator for the entropy weighted delta autocorrelation $\mathbf{R}_{E}\left(\mathbf{w}_{k}\right)$ and the entropy weighted delta crosscorrelation $\mathbf{P}_{E}\left(\mathbf{w}_{k}\right)$ of the entropy in (11) and (12), we obtain a recursive formula to update their estimates when a new sample is acquired. When a new sample arrives, $\mathbf{R}_{E}\left(\mathbf{w}_{k}\right)$ and $\mathbf{P}_{E}\left(\mathbf{w}_{k}\right)$ are modified by using the new input-output sample pair $\{\mathbf{u}(k+1), d(k+1)\}$ as

$$
\begin{aligned}
\mathbf{R}_{E}\left(\mathbf{w}_{k+1}\right)= & \frac{1}{(k+1)^{2}} \sum_{i=1}^{k+1} \sum_{j=1}^{k+1} K_{\sigma \sqrt{2}}\left(e_{j}-e_{i}\right) \cdot\left[\mathbf{u}_{j}-\mathbf{u}_{i}\right] \cdot\left[\mathbf{u}_{j}-\mathbf{u}_{i}\right]^{T} \\
= & \frac{k^{2}}{(k+1)^{2}} \mathbf{R}_{E}\left(\mathbf{w}_{k}\right) \\
& +\frac{2}{(k+1)^{2}}\left[\sum_{i=1}^{k} K_{\sigma \sqrt{2}}\left(e_{k+1}-e_{i}\right) \cdot\left[\mathbf{u}_{k+1}-\mathbf{u}_{i}\right] \cdot\left[\mathbf{u}_{k+1}-\mathbf{u}_{i}\right]^{T}\right] \\
\mathbf{P}_{E}\left(\mathbf{w}_{k+1}\right)= & \frac{1}{(k+1)^{2}} \sum_{i=1}^{k+1} \sum_{j=1}^{k+1} K_{\sigma \sqrt{2}}\left(e_{j}-e_{i}\right) \cdot\left[d_{j}-d_{i}\right] \cdot\left[\mathbf{u}_{j}-\mathbf{u}_{i}\right] \\
= & \frac{k^{2}}{(k+1)^{2}} \mathbf{P}_{E}\left(\mathbf{w}_{k}\right) \\
& +\frac{2}{(k+1)^{2}}\left[\sum_{i=1}^{k} K_{\sigma \sqrt{2}}\left(e_{k+1}-e_{i}\right) \cdot\left[d_{k+1}-d_{i}\right] \cdot\left[\mathbf{u}_{k+1}-\mathbf{u}_{i}\right]\right]
\end{aligned}
$$

This exact recursion is useful for estimating the entropy weighted delta autocorrelation and crosscorrelation of stationary signals however it is not suitable for nonstationary environments due to its increasing memory depth,. Therefore, a forgetting recursive estimator is necessary to serve satisfactorily in such situations.

$$
\begin{aligned}
\overline{\mathbf{R}}_{E}\left(\mathbf{w}_{k+1}\right) & =(1-\lambda) \overline{\mathbf{R}}_{E}\left(\mathbf{w}_{k}\right) \\
+ & \frac{\lambda}{L} \sum_{i=k-L+1}^{k} K_{\sigma \sqrt{2}}\left(e_{k+1}-e_{i}\right) \cdot\left[\mathbf{u}_{k+1}-\mathbf{u}_{i}\right] \cdot\left[\mathbf{u}_{k+1}-\mathbf{u}_{i}\right]^{T} \\
\overline{\mathbf{P}}_{E}\left(\mathbf{w}_{k+1}\right) & =(1-\lambda) \overline{\mathbf{P}}_{E}\left(\mathbf{w}_{k}\right) \\
& +\frac{\lambda}{L} \sum_{i=k-L+1}^{k} K_{\sigma \sqrt{2}}\left(e_{k+1}-e_{i}\right) \cdot\left[d_{k+1}-d_{i}\right] \cdot\left[\mathbf{u}_{k+1}-\mathbf{u}_{i}\right]
\end{aligned}
$$

\section{Outline of the Fixed-Point MEE Algorithm}

1. Initialize randomly the vector $\mathbf{w}_{0} \in \mathfrak{R}^{M \times 1}$.

2. Choose one of the two following procedure after we design both $\mathrm{M}$ and $\mathrm{L}$, where $\mathrm{M}$ is the filter order and $\mathrm{L}$ is the window length.

If $M \leq L$,

Initialize randomly the matrix $\overline{\mathbf{R}}_{E}\left(\mathbf{w}_{0}\right) \in \mathfrak{R}^{M \times M}$. else

Initialize randomly the matrix $\overline{\mathbf{\Phi}}_{E}\left(\mathbf{w}_{0}\right) \in \mathfrak{R}^{M \times M}$.

3. Initialize randomly the vector $\overline{\mathbf{P}}_{E}\left(\mathbf{w}_{0}\right) \in \Re^{M \times 1}$.

4. Update $\overline{\mathbf{R}}_{E}\left(\mathbf{w}_{k}\right)^{-1}$ or $\overline{\mathbf{\Phi}}\left(\mathbf{w}_{k}\right)$.

$$
\text { If } M \leq L \text {, }
$$

a) Update $\overline{\mathbf{R}}_{E}\left(\mathbf{w}_{k}\right)$ using (16).

b) Compute the inverse of the matrix $\overline{\mathbf{R}}_{E}\left(\mathbf{w}_{k}\right)$ else

$\left.\mathrm{a}^{*}\right)$ Compute the inverse of the matrix,

$$
\mathbf{I}_{L \times L}+\frac{\lambda}{(1-\lambda) L} \boldsymbol{\Pi}^{T} \overline{\mathbf{\Phi}}\left(\mathbf{w}_{k-1}\right) \boldsymbol{\Pi}
$$

$\left.\mathrm{b}^{*}\right)$ Update $\overline{\boldsymbol{\Phi}}\left(\mathbf{w}_{k}\right)$ using (20).

5. Update $\overline{\mathbf{P}}_{E}\left(\mathbf{w}_{k}\right)$ using (17).

6. Update the weight vector as $\mathbf{w}_{k+1}=\overline{\mathbf{R}}_{E}\left(\mathbf{w}_{k}\right)^{-1} \overline{\mathbf{P}}_{E}\left(\mathbf{w}_{k}\right)$ or

$\mathbf{w}_{k+1}=\overline{\mathbf{\Phi}}\left(\mathbf{w}_{k}\right) \overline{\mathbf{P}}_{E}\left(\mathbf{w}_{k}\right)$.

7. Go back to step 4 and repeat until convergence is reached.

Fig.1. Procedure of the recursive Fixed-Point MEE.

where the parameters $\lambda$ and $L$ are called the forgetting factor and window length. These free design parameters have an effect on the convergence properties of this recursive estimator. The practical importance of this recursive estimator is that it reduces the computational complexity from $O\left(N^{2}\right)$ to $O(L)$. This is a drastic reduction in the computation power requirements.

The weight updates by equation (10) is costly both computationally and memory-wise. This is because it requires an inversion of a $M \times M$ coefficient matrix, $\overline{\mathbf{R}}_{E}\left(\mathbf{w}_{k}\right)$ where $M$ is the filter order, at all time instants. This matrix inversion requires $O\left(M^{3}\right)$ operations. First, we need to convert the summation of the rank-one update (16) to the following

$$
\overline{\mathbf{R}}_{E}(\mathbf{w}(k+1))=(1-\lambda) \overline{\mathbf{R}}_{E}(\mathbf{w}(k))+\frac{\lambda}{L} \boldsymbol{\Pi} \cdot \boldsymbol{\Pi}^{T}
$$

where $\boldsymbol{\Pi}=\left[\begin{array}{llll}\mathbf{q}_{k} & \mathbf{q}_{k-1} & \ldots & \mathbf{q}_{k-L+1}\end{array}\right], \mathbf{q}_{i}=\sqrt{K_{\sigma \sqrt{2}}\left(e_{k+1}-e_{i}\right)} \cdot\left(\mathbf{u}_{k+1}-\mathbf{u}_{i}\right)$. Then, by using the matrix inversion lemma

$$
(\mathbf{A}+\mathbf{B C D})^{-1}=\mathbf{A}^{-1}-\mathbf{A}^{-1} \mathbf{B}\left(\mathbf{C}^{-1}+\mathbf{D A}^{-1} \mathbf{B}\right)^{-1} \mathbf{D} \mathbf{A}^{-1}
$$




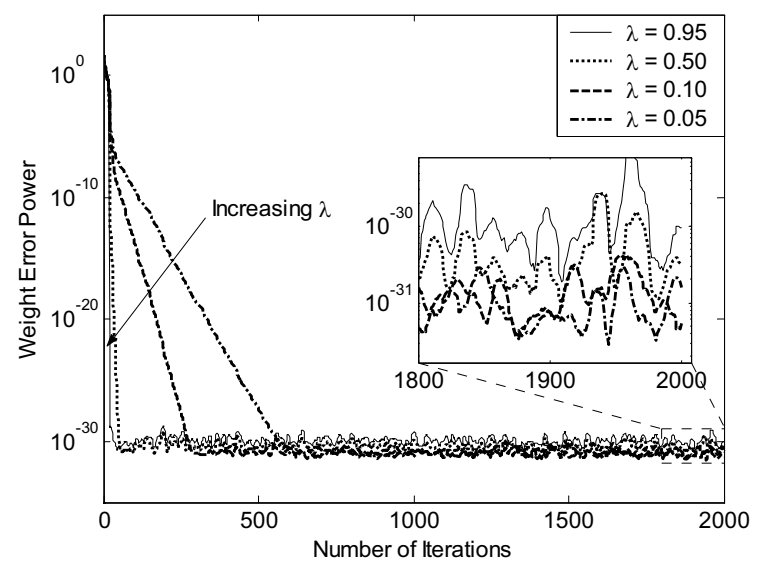

Fig.2. Comparison of the convergence properties of the recursive Fixed-Point MEE for MA(9) with different values of the forgetting factor.

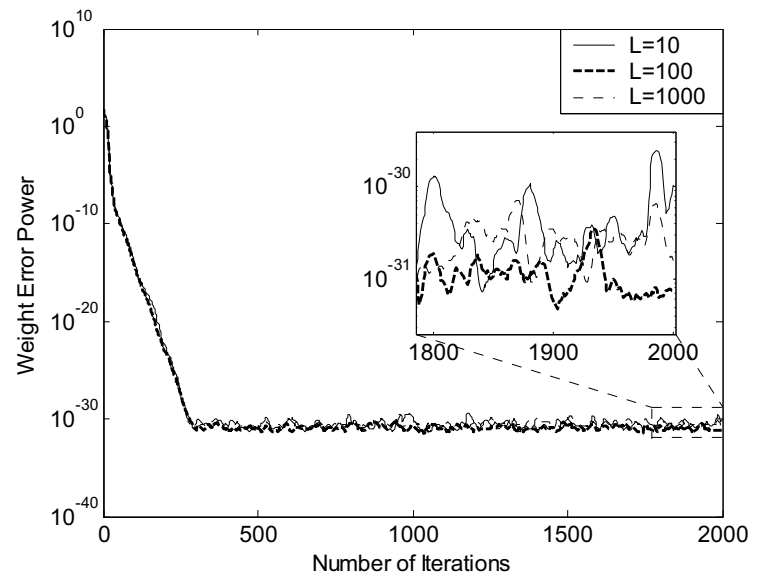

Fig.3. Comparison of the convergence properties of the recursive Fixed-Point MEE for MA(9) with different values of the window length.

with $\mathbf{A}=(1-\lambda) \overline{\mathbf{R}}_{E}\left(\mathbf{w}_{k}\right), \mathbf{B}=\frac{\lambda}{L} \boldsymbol{\Pi}, \mathbf{C}=\mathbf{I}$, and $\mathbf{D}=\boldsymbol{\Pi}$, we obtain a recursive inverse autocorrelation of the entropy

$$
\begin{aligned}
\left(\left[\overline{\mathbf{R}}_{E}\left(\mathbf{w}_{k}\right)\right]^{-1}\right. & \left.=\overline{\boldsymbol{\Phi}}\left(\mathbf{w}_{k}\right)\right), \\
\overline{\boldsymbol{\Phi}}\left(\mathbf{w}_{k+1}\right) & =\frac{1}{1-\lambda} \overline{\boldsymbol{\Phi}}\left(\mathbf{w}_{k}\right) \\
& -\frac{\lambda}{(1-\lambda)^{2} L} \overline{\boldsymbol{\Phi}}\left(\mathbf{w}_{k}\right) \boldsymbol{\Pi}\left(\mathbf{I}_{L \times L}+\frac{\lambda}{(1-\lambda) L} \Pi^{T} \overline{\boldsymbol{\Phi}}\left(\mathbf{w}_{k}\right) \boldsymbol{\Pi}\right)^{-1} \boldsymbol{\Pi}^{T} \overline{\boldsymbol{\Phi}}\left(\mathbf{w}_{k}\right) .
\end{aligned}
$$

That matrix inversion included in (20) requires $O\left(L^{3}\right)$ operations where $L$ is the window length.

\section{SIMULATIONS}

In the simulations, we will investigate the effect of the forgetting factor, the window length, and the kernel size on

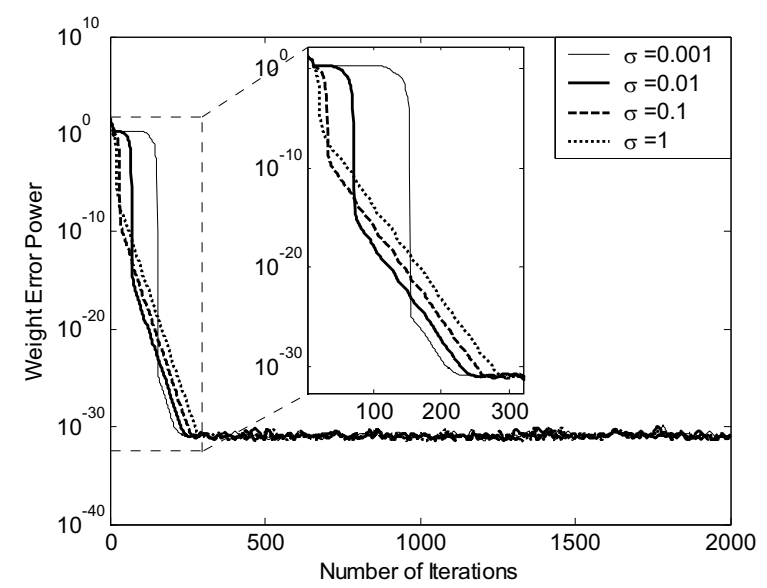

Fig.4. Comparison of the convergence properties of the recursive Fixed-Point MEE for MA(9) with different values of the kernel size.

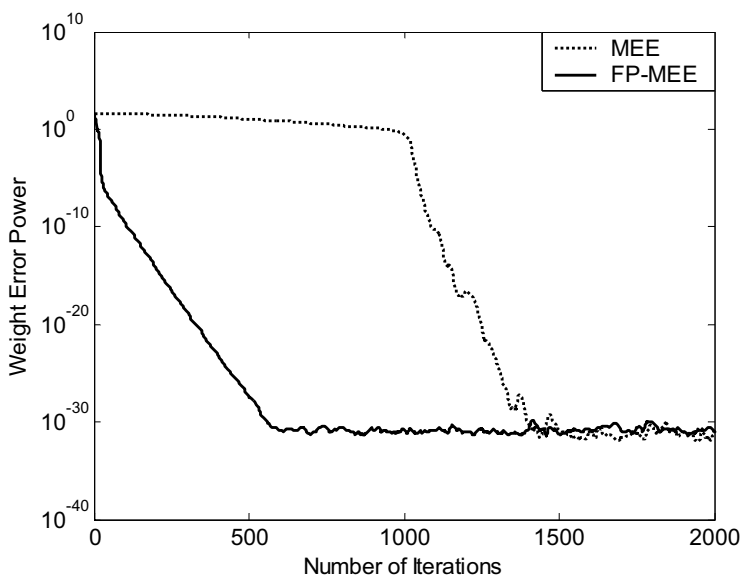

Fig.5. Comparison of the convergence speed of the recursive MEE and recursive Fixed-Point MEE for MA(9)

the convergence speed and the convergence accuracy (variance after convergence) of the newly introduced recursive Fixed-Point MEE. Also, we will experimentally verify the different adaptation performance of the recursive Fixed-Point MEE with respect to the recursive MEE. Simulations are particularly useful in this case because the differences between the two algorithms are not as easy to translate to practical measures of convergence rate and misadjustment as in the LMS case. We consider two kinds of plant identification models, a moving-average model (MA), and an autoregressive model (AR). The adaptive weights were initialized randomly for each case. Further, in order to make the result independent of the input and weight initializations, we perform Monte-Carlo simulations with 100 different inputs and 100 different weight initializations for each input. 


\subsection{Example 1: MA(9)}

In the first case study, we consider a moving-average model with transfer function given by (order=9) [10]

$$
\begin{aligned}
H(z)=0.1 & +0.2 z^{-1}+0.3 z^{-2}+0.4 z^{-3}+0.5 z^{-4} \\
& +0.4 z^{-5}+0.3 z^{-6}+0.2 z^{-7}+0.1 z^{-8} .
\end{aligned}
$$

The FIR adaptive filter is selected with equal order (Model Order $=9$ ). The input to both the plant and the adaptive filter is white Gaussian noise with unit power. The objective is to adapt the weights $\mathbf{w}$ of an FIR filter so as to emulate the plant as closely as possible. A standard method of comparing the performance in system identification problems is by plotting the weight error norm since this is directly related to misadjustment. In this case ideally, we can exactly track the output of the plant. Although entropy cost functions are designed for identification of nonlinear systems with nonlinear filters, here we are interested in the adaptation performance only, so we chose a very simple linear model and plant to obtain a convex cost function.

In the first simulation, we investigate the effect of the forgetting factor on the convergence time and the convergence accuracy (variance after convergence) of the recursive Fixed-Point MEE. For this purpose, we have utilized this recursion for 2000 iterations. Four different values are used for the forgetting factor: $0.95,0.5,0.1$, and 0.05 . The convergence plots of the estimates are shown in Fig.2. Starting from the same initial estimate, the three recursions converge after approximately 30, 50, 300 and 600 iterations. As expected, the faster the convergence, the larger the estimation variance is. When we evaluate the variances of the estimated values over the last 200 samples of each convergence curve, we see that larger forgetting factors result in larger variance; the variances (means) are respectively, $2.85 \times 10^{-60}\left(1.51 \times 10^{-30}\right), 2.89 \times 10^{-61}\left(5.09 \times 10^{-31}\right)$, $9.24 \times 10^{-63}\left(1.41 \times 10^{-31}\right)$, and $4.43 \times 10^{-63}\left(1.03 \times 10^{-31}\right)$ In these runs, we have used $\mathrm{L}=100$ and sigma $=0.707$. This result conforms to the well-known general behavior of the forgetting factor in recursive estimates. This is an intrinsic trade-off between speed and variance, which the designer must consider in selecting the forgetting factor.

The second simulation studies the effect of the window length, which approximates the expectation operator. For this purpose, we have fixed the forgetting factor to $\lambda=0.1$, and the kernel size to $\sigma=0.707$. Three values of $L$ are tried: 10, 100, and 1000. The results of the recursive FixedPoint MEE using these three different window lengths are shown in Fig.3. As expected, the speed of convergence is not affected by the variations in this parameter. Only, the estimation variance after convergence is a little affected. Specifically, the variances (means) of the estimates for these three cases over the last 200 iterations of the recursion are
$2.34 \times 10^{-61}\left(4.59 \times 10^{-31}\right), \quad 1.07 \times 10^{-62}\left(1.14 \times 10^{-31}\right)$, and $8.84 \times 10^{-63}\left(2.95 \times 10^{-31}\right)$. This conforms to the general behavior of the sample mean approximation for expectation: The more samples used, the smaller the variance gets. The trade-off in the selection of this parameter is between the accuracy after convergence and the memory requirement. The larger $L$ gets, the more storage space is required for previous samples in memory; on the other hand, estimation variance is decreased.

This third simulation investigates the effect of kernel size on the convergence performance of the recursive FixedPoint MEE. As we know, Parzen windowing has a bias that increases with larger kernel sizes, whereas its variance increases with smaller kernel sizes. The convergence plots of the recursions for various values of the kernel size are shown in Fig.4. In all runs, the forgetting factor was fixed to 0.1 and the window length was taken as 100 . For the Gaussian kernel function with sizes of $0.001,0.01,0.1$ and 1 , the variances (means) over the last 200 samples of the recursions turned out to be $4.34 \times 10^{-63}\left(1.46 \times 10^{-31}\right)$, $4.68 \times 10^{-63}\left(1.21 \times 10^{-31}\right), \quad 2.64 \times 10^{-63}\left(1.17 \times 10^{-31}\right) \quad$ and $3.89 \times 10^{-63}\left(1.26 \times 10^{-31}\right)$. The speed and accuracy of convergence is not affected by the variations of the kernel size for this example. On the other hand, as can be observed in the enlarged plot, the smaller the kernel size the flatter is the slope of the weight error curve at the origin, followed by a sharp drop before convergence.

The purpose of the fourth simulation is to show the faster convergence of the recursive Fixed-Point MEE compared to the recursive MEE for the same misadjustment. Fig.5 shows the plot of the weight error norm. The weight misadjustment values for the last 200 samples of error are $1.31 \times 10^{-31}\left(3.95 \times 10^{-62}\right)$ and $1.85 \times 10^{-31}\left(2.24 \times 10^{-62}\right)$ for the recursive MEE and the recursive Fixed-Point MEE respectively (For practical purposes, we consider misadjustment values as zero for values less than $10^{-30}$ ). Thus with the same misadjustment values, it can be observed that Fixed-Point MEE converges in 600 iterations whereas MEE takes 1500 iterations to converge.

\subsection{Example 2: AR(1)}

As a second case study, we select for the unknown plant a first order autoregressive model,

$$
H_{2}(z)=\frac{1}{1-0.9 z^{-1}} .
$$

The unknown system $H_{2}(z)$ is approximated by a moving average model with 16 taps. The white Gaussian noise inputs with unit variance are applied to both the plant and the adaptive filter. We have set the window length to 100 and the kernel size to 0.707 . Also, we have fixed the 


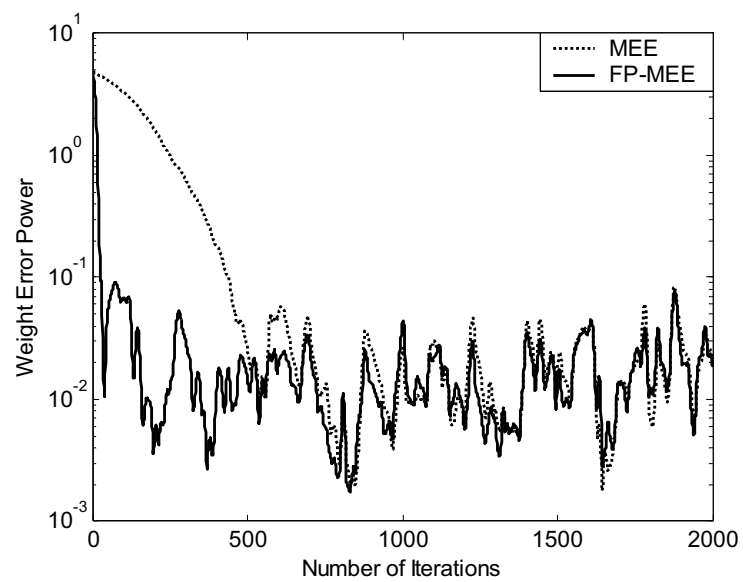

Fig.6. Comparison of the convergence speed of the recursive MEE and recursive Fixed-Point MEE for AM(1)

forgetting factor of MEE and Fixed-Point MEE to 0.4 and 0.05 , respectively.

Fig.6. shows the best results for MEE and Fixed-Point MEE of the average weight error power to identify the first order autoregressive model. We can observe that the recursive Fixed-Point MEE takes just 35 iterations to converge with a misadjustment of 0.0248 as compared to the recursive MEE which takes nearly 500 iterations with misadjustment of 0.0254 . This result shows that even when the unknown plant is an AR model, the recursive FixedPoint MEE converges faster than the recursive MEE based on the gradient algorithms.

\section{CONCLUSION}

We have presented a new algorithm, the Fixed-Point Minimum Error Entropy by using the first order optimality condition of the error entropy and the fixed-point iteration. This update rule is analogous to the RLS update rule that tracks the Wiener solution with every update. Also, we have derived the recursive formulations to extend the estimation flexibility of the Fixed-Point MEE. This recursive FixedPoint MEE allows on-line treatment of signals for computationally simple and fast manipulation of the adaptive system parameters.

We have investigated the effect of the forgetting factor, the window length, and the kernel size on the convergence properties of the Fixed-Point MEE in simulations. Increased forgetting factor resulted in faster convergence, smaller misadjustment and larger variance. Increased window length had no effect on convergence speed and larger variance. Increased kernel size had no effect on convergence speed and accuracy, but resulted in a weight error curve that descended more sharply and then by a gradual slope to the final value.
Finally, we tested the performance of the recursive MEE and the recursive Fixed-Point MEE on the adaptation of FIR filter for MA(9) and AR(1) system. We conclude that the recursive Fixed-Point MEE converges much faster than the recursive MEE. However, the complexity of the fixed point MEE is much higher than that of the recursive MEE using a gradient approach. However, in applications where speed is crucial, the enhanced convergence speed may be preferable.

There are important aspects that need further investigation. The first is the proof of convergence. Although during all our simulations the Fixed Point MEE was always stable, there is no theoretical proof of convergence. The other aspect is to try to simplify the updates of the inverse of the entropy weighted delta autocorrelation function. The determining term is the product $D A^{-1} B$, which is $L \times L$ dimension. We need to design $L$ as a compromise between accuracy and complexity.

Acknowledgments: This work was partially supported by ECS-0300340 and ECS-0601271. We thank the anonymous reviewers for their constructive suggestions which will be useful in further investigating this novel algorithm.

\section{REFERENCES}

[1] J.C. Principe, D. Xu and J. Fisher, "Information Theoretic Learning," in S. Haykin, Unsupervised Adaptive Filtering, Wiley, Newyork, vol I, pp. 265-319, 2000.

[2] D. Erdogmus, "Information Theorectic Learning: Renyi's Entropy and its Applications to Adaptive System Training," Ph.D Dissertation, University of Florida, Gainesville, FL, 2002.

[3] D. Erdogmus, J.C. Principe, "An Entropy Minimization algorithm for Supervised Training of Nonlinear Systems," IEEE trans. of Signal Processing, vol.50, no.7, pp. 1780-1786, July 2002.

[4] A. Chicocki and S.I. Amari, Adaptive Blind Signal and Image Processing. New York: Wiley, 2002.

[5] P.A. Reglia and E. Kofidis, "Monotonic convergence of fixedpoint algorithms for ICA," IEEE Trans. Neural Networks., vol. 14, pp. 943-949, July 2003.

[6] D. Erdogmus, J.C. Principe, S.P. Kim, J.C. Sanchez, "ARecursive Renyi's Entropy Estimator," Porc. NNSP'02., pp.209-217, Martigny, Switzerland, 2002.

[7] D. Erdogmus, J.C. Principe, K.E. Hild II, "On-Line Entropy Manipulation: Stochastic Information Gradient," IEEE Signal Processing Letters, vol. 10, no.8, pp. 242-245, Aug. 2003.

[8] Simon Haykin, Adaptive Filter Theory, Prentice Hall, Upper Saddle River, $4^{\text {th }}$ edition, 2001.

[9] J.K. Hale, Ordinary Differential Equations, John Wiley and Sons, New York, 1969.

[10] E. Walach and B. Widrow, "The Least Mean Fourth(LMF) Adaptive Algorithm and its Family," IEEE trans. of Inf. Theory, vol. IT 30, No.2, pp. 275-283, March 1984 